\title{
Dynamiques Sociales Et Gestion Fonciere En Zone Cotonniere Du Mali
}

\section{Baba Coulibaly}

Département de Géographie, Faculté d'Histoire et de Géographie, Université des Sciences Sociales et de Gestion de Bamako, Mali

\section{Souleymane Sidi Traoré Cheick Hamalla Diakité}

Unité Système d’Information Géographique et Télédétection, Labosep, CRRA de Sotuba, IER, Bamako, Mali

\section{Abdou Ballo}

Département de Géographie, Faculté d'Histoire et de Géographie, Université des Sciences Sociales et de Gestion de Bamako, Mali

\section{Alou Traoré \\ Moriké Diawara}

Unité Système d’Information Géographique et Télédétection, Labosep, CRRA de Sotuba, IER, Bamako, Mali

\section{Sidi Dembélé}

Département de Géographie, Faculté d'Histoire et de Géographie, Université des Sciences Sociales et de Gestion de Bamako, Mali

\begin{abstract}
In Mali the management of rural area in the cotton belt has become more complex. It presents social, economic, ecological and cultural issues. Indeed, the increasing numbers of actors due to migration and population growth, as well as the emergence of new agricultural systems have created new dynamics in terms of companies and natural resources management. This work aimed at analysing the functioning of territories and actors through their social organisation and resource management strategies in the villages of Nafègué, Benguéné and Ziguéna. The methodology used was based on direct observation and qualitative surveys with several categories of actors identified using a diagnostic tools. The results show that the management of associations and cooperatives is identical in all the three villages. They are in principle independent from each other and have a freedom of action. However, there are mutual aid relations between the different associations. At village scale, land management follows similar
\end{abstract}


principles. The land is under the responsibility of the village head, but there are often land owners. Nowadays, because of land pressure resulting from population growth and agricultural activities, land issue has become one of the biggest concern land access rules more complex. These facts require new collective processes for natural resource management.

Keywords: Space, territorial management, actors, natural resources, land

\section{Résumé}

$\mathrm{Au}$ Mali la gestion de l'espace rural dans la zone cotonnière s'est complexifiée. Elle présente des enjeux d'ordre social, économique, écologique et culturel. En effet l'amélioration des conditions de production due à l'émergence de nouveaux systèmes agraires, la croissance démographique, la multiplication des acteurs et les migrations ont créé de nouvelles dynamiques en matière de gestion des sociétés et des ressources naturelles. Ce travail visait à analyser le fonctionnement des territoires à travers l'organisation sociale des acteurs et les modes de gestion des ressources foncières dans les villages de Nafègué, Benguéné et Ziguéna. La méthodologie utilisée s'est basée sur l'observation directe et des enquêtes qualitatives avec plusieurs catégories d'acteurs à partir d'outils de diagnostic. Les résultats montrent que le fonctionnement des différentes associations et coopératives est identique dans les trois villages. Elles sont en principe indépendantes les unes des autres et disposent d'une liberté d'action. Cependant, il existe des relations de mutualité et d'entraide entre les différentes associations. A l'échelle de chacun des villages la gestion foncière obéit à des principes similaires. La terre est placée sous la responsabilité du chef de village, mais il existe souvent des propriétaires terriens. De nos jours, à cause de la pression foncière liée à la surexploitation agricole des terres, l'augmentation de la population et du cheptel, la question foncière est l'une des plus préoccupantes et les règles d'accès se complexifient davantage. Ces réalités majeures imposent l'initiation et le développement de nouveaux processus collectifs de gestion des ressources naturelles.

Mots-clefs: Espace, gestion territoriale, acteurs, ressources naturelles, foncier

\section{Introduction}

Depuis près de deux décennies, avec l'avènement de la démocratie et de la décentralisation, les Etats africains ont connu une promotion de la société civile. Selon Canet et Audet (2005), l'irruption de la société civile transnationale sur la scène mondiale a nourri l'espoir d'une véritable 
inflexion du cours de la globalisation néolibérale. Au Mali, la fin de la deuxième République et l'avènement de la démocratie portée par les acteurs politiques et ceux de la société civile a ouvert la voie à un nouveau mode de gestion territoriale susceptible d'assurer une meilleure prise en compte des besoins et des priorités exprimées par les populations, et pour ce faire de renforcer le rôle et la place des acteurs locaux. Dans une telle perspective, il s'agit tout à la fois de permettre et de favoriser la concertation et la collaboration entre des acteurs économiques, sociaux et publics et de susciter l'émergence de nouveaux acteurs. Cela permettra la participation active des bénéficiaires à l'élaboration et à la mise en œuvre des programmes d'action qui les concernent. Les sociétés paysannes entretiennent directement des relations avec le vivant et avec le territoire, elles semblent, par nature, désignées à être des acteurs déterminants du développement durable (Aubertin et Pinto 2006). C'est grâce à la détermination des populations locales elles-mêmes et aux efforts soutenus de leurs organisations que, depuis une vingtaine d'années, leur ascension est remarquable sur la scène politique, économique, sociale, culturelle et démocratique (Moulaye, 2005). Ainsi la problématique de l'implication et de la participation des populations dans les processus décisionnels se pose aujourd'hui comme l'un des nouveaux enjeux de la poursuite de la décentralisation au Mali.

Les villages de Benguéné (Cercle de Bla), de Ziguéna (Cercle de Sikasso) et de Nafègué (Cercle de Kadiolo), où la présente étude a été conduite (Figure 1), renferment des organisations sociales (Coopératives et associations). Celles-ci sont considérées, selon les termes de la loi 01-076 du 28 juin 2001 régissant les associations et coopératives au Mali, comme des sociétés de personnes de type particulier fondées sur les principes d'union, de solidarité et d'entraide mutuelle. Leurs membres se regroupent volontairement pour atteindre un but de développement économique et social commun par la constitution d'une entreprise qu'ils gèrent démocratiquement à leurs avantages et/ou à leurs risques communs et au fonctionnement de laquelle ils s'engagent à participer activement. Pour Leroy et al. (2006), la gestion communautaire, décentralisée et participative constitue aujourd'hui le maitre-mot des politiques d'environnement, et les communautés locales sont non seulement officiellement conviées à gérer les ressources naturelles, mais sont aussi considérées comme les principales garantes d'une gestion durable. Ces organisations nouent des relations complexes notamment dans le cadre de la gestion des ressources naturelles et des actions de développement. Mais il se trouve que les ressources foncières des trois villages présentent aujourd'hui des enjeux importants liés à multiples facteurs. D'un côté la zone est sous influence d'une forte pression due à une croissance démographique continue avec ses corollaires, et de l'autre on assiste à une marchandisation croissante de la terre et une compétition accrue 
entre différents acteurs. L'objectif de cet article est d'analyser le fonctionnement des terroirs de ces trois villages à travers l'organisation sociale des acteurs et les modes de gestion des ressources. L'article tente dans un premier temps de comprendre le rôle des organisations sociales dans le processus de développement et leurs relations; dans un second temps, il examine les logiques de gestion foncière à travers l'accès à la terre, les enjeux et les risques de litiges liés à cette ressource.

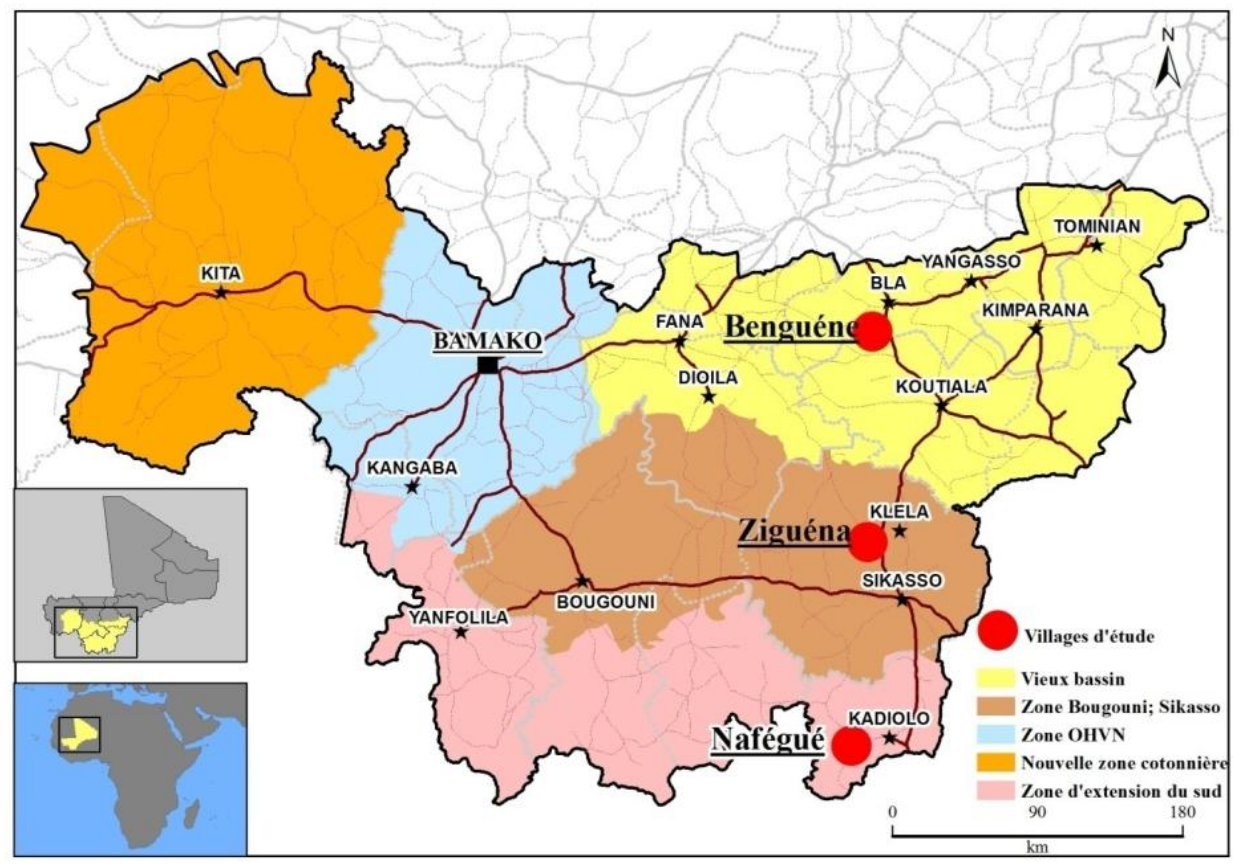

Figure 1: Localisation des villages d’études

\section{Méthodologie}

La méthodologie utilisée s'est basée sur des enquêtes qualitatives avec plusieurs catégories d'acteurs et l'observation directe. La recherche a été menée dans le cadre de la mise en œuvre du volet RechercheDéveloppement du projet PASE II (Amélioration de la productivité, de la durabilité et de la compétitivité des systèmes d'exploitation de la zone cotonnière). Basé sur une démarche de Recherche Action en Partenariat (RAP), le PASE II avait déjà créé des cadres de concertation représentatifs de toutes les catégories socioéconomiques dans les trois villages-sites pour faciliter ses activités. En conséquence, nos investigations ont été rendues faciles notamment en ce qui concerne la constitution des focus groupes. Dans chacun des villages, nos activités ont commencé par des discussions avec le cadre de concertation dont la taille varie entre 10 et 20 personnes. Ensuite les entretiens individuels ont continué avec des personnes ressources, tantôt résidant dans le village (personnel enseignant, agent de santé, responsables 
d'associations villageoises), tantôt ne résidant pas toujours dans le village (agent d'encadrement de la Compagnie Malienne de Développement Textile, autorités administratives). Le choix de ces interlocuteurs a été opéré en fonction de leur disponibilité sur leur lieu de travail (école, centre de santé) ou à la maison. Ainsi les Directeurs d'école, les enseignants qui ont le plus duré dans les villages, les médecins ou Directeurs techniques des centres de santé communautaires (CSCOM), étaient privilégiés. En plus des interviews avec les cadres de concertation, 20 entretiens individuels, soit environ sept personnes par village, ont été réalisés.

Aussi, dans chaque village des entretiens informels ont été réalisés concomitamment avec les opérations d'observation. Les unités d'observation portaient sur les rassemblements des personnes âgées sous l'arbre à palabre, les regroupements de jeunes $\left(\right.$ grins $\left.^{9}\right)$, les relations entre les individus au sein des villages. Les missions d'enquêtes ont été réalisées entre octobre 2014 et juin 2015, soit une période de 09 mois. Ces entretiens et discussions se sont appuyés sur des outils de diagnostic: guide d'entretien de diagnostic villageois, diagramme de Venn). Les informations collectées ont été d’abord traduites avant de faire ensuite l'objet d'une analyse de contenu selon la méthode de Walin, (2007).

\section{Résultats et Discussion}

Organisations sociales et leurs relations

Les trois villages sont organisés autour de plusieurs organisations et coopératives socioprofessionnelles (Tableau1). Leur fonctionnement est identique dans chacun des villages.

Tableau 1: Associations et coopératives existantes dans les trois villages

\begin{tabular}{|c|c|}
\hline Noms de villages & Noms des associations et coopératives \\
\hline \multirow{3}{*}{ Nafègué } & $\begin{array}{l}\text { - Coopérative des Producteurs de coton } \\
\text { (C.P.C) }\end{array}$ \\
\hline & $\begin{array}{l}\text { Benkadi, Alla ka Chidiman, et } \\
\text { Kotognongotala }\end{array}$ \\
\hline & $\begin{array}{ll}- & \text { Association des jeunes } \\
\text { - } & \text { Association des Minianka }\end{array}$ \\
\hline Benguéné & $\begin{array}{ll} & \text { Coopérative des Producteurs de Coton (CPC) } \\
\text { - } & \text { Association Benkadi des femmes } \\
\text { - } & \text { Association Faso Niéta ton des éleveurs } \\
\text { - } & \text { Association des jeunes }\end{array}$ \\
\hline Ziguéna & $\begin{array}{ll}\bullet & \text { Coopérative des Producteurs de coton (CPC) } \\
\bullet & \text { Gouantan (Jèkadi) } \\
\text { - } & \text { Benkadi } \\
\text { - } & \text { Katienepi (Kaniuma kè) } \\
\end{array}$ \\
\hline
\end{tabular}

${ }^{9}$ Lieu de rencontre, de distraction, d'échanges, où les jeunes de même génération et même les adultes, se réunissent autour du thé et passer le temps. 
Elles sont par principe indépendantes les unes des autres et disposent d'une liberté d'action. Cependant, dans le cadre du développement local, ces organisations sont obligées d'œuvrer ensemble.

Les trois villages disposent chacun d'une Coopérative des Producteurs de Coton (CPC). Celles-ci poursuivent les mêmes objectifs partout. Il s'agit notamment de promouvoir la culture du coton. Les CPC assurent également les questions relatives à l'approvisionnement des paysans en engrais, le magasinage des produits, la formation des paysans, etc.

A Nafègué, en dehors de cette organisation en commun, il existe 03 groupements de femmes qui sont: Benkadi, Alla $\mathrm{ka}$ Chidiman, et Kotognongotala. Ils mènent généralement des activités rémunérées comme la mise en valeur des champs collectifs de coton, d'arachide et de riz. Ces activités champêtres permettent de renflouer leur caisse. Concernant la jeunesse, en dehors des regroupements au sein des groupes d'âge, elle se retrouve dans une grande association qui couvre tout le village. Quant à l'association des Minianka, elle a pour objectif de promouvoir l'entraide au sein de leur communauté ethnique. A Ziguéna, il existe plusieurs organisations sociales comme Gouantan (jèkadi) et Benkadi des producteurs de pomme de terre, Katienepi (kaniuma kè) des producteurs de riz. Ce sont des associations basées sur l'agriculture. Elles œuvrent en particulier pour la promotion du riz, de la pomme de terre, disposent toutes de leur récépissé et s'entraident mutuellement.

L’association Benkadi des femmes de Benguéné existe depuis 2003 et compte actuellement plus de 200 adhérentes. Au même titre que dans les villages ci-dessus cités, les femmes de Benguéné mènent des activités comme la récolte de coton, de mil en vue d'alimenter la caisse de leur association. Elles ont été également initiées à l'embouche de petits ruminants grâce à l'appui du Projet Agro Écologie. Les ressources obtenues à partir de ces activités sont destinées à payer les quotes-parts en cas de panne de leur plate-forme multifonctionnelle. Quant à Faso nièta ton des éleveurs, il a pour objectif, à travers la promotion de l'élevage de lutter contre la paupérisation croissante des paysans et l'exode rural. L'élevage devient ainsi une activité complémentaire intéressante qui va occuper la jeunesse pendant la saison sèche. Ce qui permet de créer des emplois et de lutter contre le départ massif des bras valides en exode après les récoltes. 


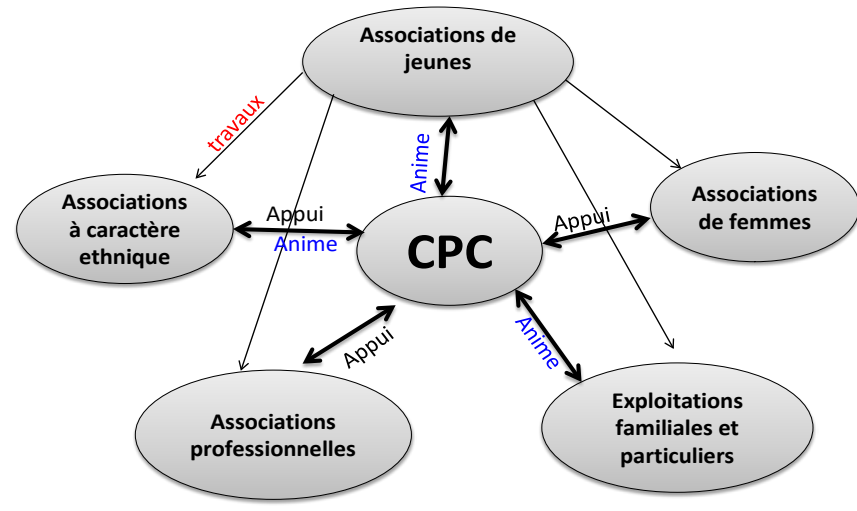

Figure 2: Schéma des relations dynamiques entre les associations et coopératives des trois villages

Il existe en effet des relations de mutualité et d'entraide entre les différentes associations (Figure 2). Au niveau des trois villages, toutes les autres organisations peuvent impliquer la jeunesse dans leurs activités. Les jeunes sont généralement à la disposition du village pour tous les travaux d'intérêt commun en termes d'investissement humain (confection de briques, assainissement ${ }^{10}$, construction des maisons notamment celles des enseignants). La jeunesse s'occupe également chaque année de l'entretien des routes ${ }^{11}$, qui sont généralement dégradées par les écoulements d'eaux pluviales, en vue d'une meilleure évacuation du coton. La jeunesse est réquisitionnée pour l'organisation des événements ou festivités de fin de saison pluvieuse à Nafègué. Les grins de jeunes disposent des équipements comme des chaises qu'ils mettent à la disposition des autres associations en cas de rassemblement et inversement. A Benguéné, les jeunes qui partent en exode rural en période de récolte du coton sont taxés à 25000 FCFA. A ce sujet, la CPC a pu collecter une somme de 350000 FCFA d'amende en 2014. Cette somme d'argent a servi à payer des tôles pour couvrir un magasin de stockage d'engrais de Benguéné.

Les CPC développent des liens forts avec les autres organisations sociales. Elles apparaissent comme les organisations mères qui appuient généralement les autres : achats de produits, récoltes, etc. En outre toutes les associations et les producteurs particuliers s'approvisionnent en engrais,

\footnotetext{
${ }^{10}$ Il s’agit notamment du désherbage de la cour des écoles et des CSCOM.

${ }^{11}$ Ce travail rassemble les jeunes chaque mercredi à Benguéné sur la routes qui mène à N’Golokouna. Ils constituent une main d'œuvre importante pour les femmes.
} 
insecticides, herbicides, pesticides, et autres intrants agricoles auprès des CPC. Elles peuvent également aider en préfinançant le déficit de remboursement des paysans qui ne sont pas arrivés à honorer leurs engagements vis-à-vis de la Compagnie Malienne de Développement Textile (CMDT).

Par ailleurs, les autres organisations sociales (associations des femmes, associations à caractère ethnique, associations professionnelles, exploitations familiales) n'ont aucun lien direct. Elles ne se retrouvent qu'à travers les actions de la jeunesse ou des CPC. Evoluant toutes en milieu rural, ces organisations sociales, de par leurs activités, sont d'une façon ou d'une autre rattachées à la terre.

Nous constatons qu'il y a une participation de plus en plus accrue des femmes dans les processus de développement. Leur participation aux différents cadres de concertation où elles partagent les mêmes espaces mais aussi la parole avec les hommes, est une avancée notoire comme le note Bertrand (2001). Les analyses en termes de " genre et développement » ont connu un développement spectaculaire depuis plus d'une décennie en Afrique subsaharienne (Bozon, Locoh (dir), 2000, Bertrand, 2001). Depuis les années 1990, bailleurs de fonds du développement, Organisations Non Gouvernementales (ONG) et associations déclinent la gestion des espaces au féminin, dans les milieux urbains autant que ruraux, selon une problématique du «rattrapage» des cadets sociaux autant que sous l'angle des complémentarités hommes/femmes (Bertrand, 2001).

\section{Gestion foncière}

Comme partout ailleurs au Mali, la gestion du foncier reste sensible dans les villages d'études. La terre étant la première ressource du monde rural, elle se trouve toujours au centre des débats. Si les populations reconnaissent que la terre appartient à l'Etat, elles jouissent tout de même des droits d'usufruit. A l'échelle de chacun des trois villages la gestion foncière obéit à des principes similaires. D’une manière générale, la terre est placée sous la responsabilité du chef de village, mais il existe en réalité des propriétaires terriens.

\section{Accès à la terre}

Au Mali, la gestion du foncier rural demeure fortement influencée par les règles coutumières, pour lesquelles la terre constitue un patrimoine communautaire. En effet la propriété foncière est généralement du ressort du groupe familial ou lignagère. L'accès (Figure 3) se fait selon les valeurs coutumières, la terre n'étant pas encore monétisée. Il est basé sur demande simple auprès des propriétaires terriens ou du chef de village. Pour Goislard et Djiré (2007) «L'oralité est au cour des accords de prêts et de dons». 


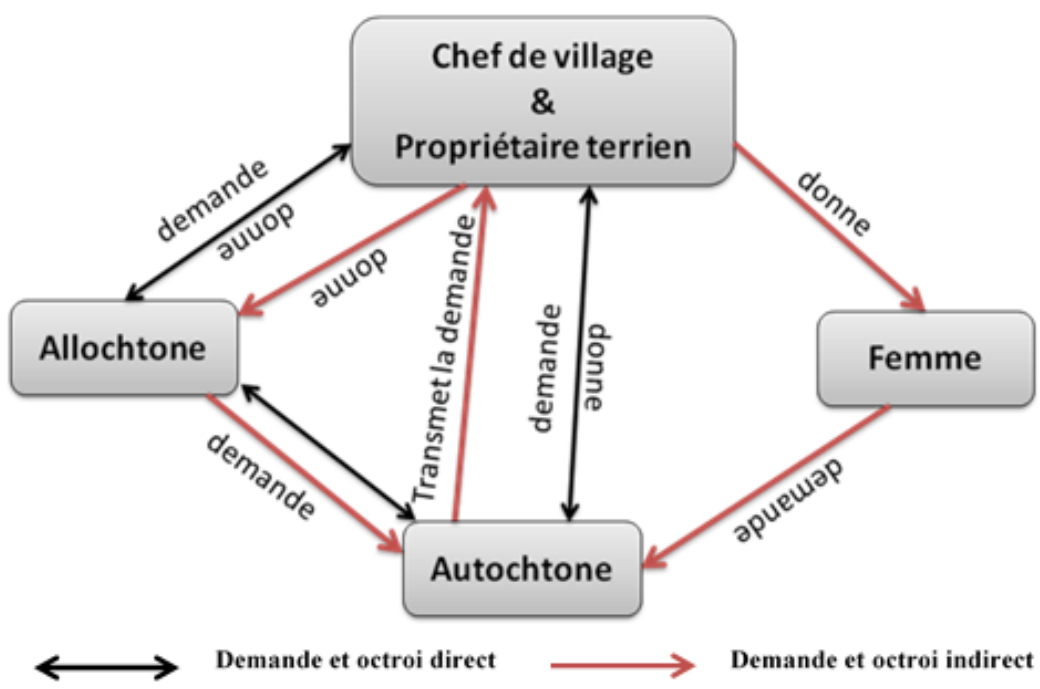

Figure 3 : Schéma des processus d'accès à la terre dans trois villages NB: dans ce schéma nous utilisons le verbe «donner » dans le sens de l'usufruit, quand on sait qu'il est admis au sein de toutes ces communautés villageoises que la terre appartient à

l'Etat.

A Benguéné, l’allochtone passe obligatoirement par le chef de village qui, à son tour, l'emmène chez le chef coutumier «le Masa » pour trancher son installation et lui attribuer de la terre. Pour des raisons d'exploitation à court terme, l'étranger peut également accéder à la terre par affinité sans passer par toutes les étapes sus-indiquées. Sur le terroir de Benguéné, il existe un espace dégagé à l'Ouest du village qui est destiné aux champs des allochtones (enseignants, agents de santé, etc.). L'allochtone qui s'installe définitivement n'est jamais servi en terre sans l'autorisation du chef de village.

A Ziguéna, même si un propriétaire terrien accorde la terre à un étranger, le chef de village est ensuite impliqué et c'est lui qui tranche en dernier ressort. Une fois que la terre est accordée, le demandeur est informé de l'ensemble des règles et principes (dugu ladaw) qui gouvernent la pratique foncière et agricole dans le village. Il y a des pratiques interdites à la culture selon les jours. "dugu lada ani taanaw bè fo i ye, n’y bè se i b'i sigi, n'y te se i bi taa" ou (l'étranger qui peut respecter les règles locales s'installe, sinon il s'en va), explique un conseiller du village. Cependant lorsque le demandeur décède, la demande doit toujours être renouvelée par ses descendants car la terre est accordée à un individu et non à une lignée. Le village de Nafègué connait les mêmes conditions d'accès à la terre que Ziguéna avec lequel il partage la même aire culturelle. 
D’une manière générale et dans les trois villages les emprunts de terres sont possibles entre autochtones, dans la mesure que l'emprunteur n'a pas le droit de planter des arbres sur la parcelle de terre empruntée. Ces transactions peuvent se faire sans l'implication du chef de village. Ziguéna fait exception à cette règle où le chef de village est toujours impliqué en vue de prévenir les problèmes dans l'avenir. Malgré tout la terre reste une ressource précieuse qui ne se vend pas encore et appartient uniquement aux hommes. Les femmes sont obligées d'utiliser leur mari et parents de sexe masculin comme intermédiaires pour accéder aux parcelles à des fins de productions céréalières ou maraîchères (Figure 3). A la question de savoir si la femme peut être propriétaire terrienne, un conseiller du chef de village de Ziguéna s'exclame : "muso dugukolo tigi, ali n'a bina kè, ah, amakè folo » ou (la femme propriétaire terrienne, ça peut être possible dans le futur, mais ce n'est pas encore le cas). A l'exception de leurs parents directs, il n'est pas encore permis qu'elles cherchent directement la terre auprès des propriétaires terriens. Bertrand, (2001) estime que la participation des femmes aux dynamiques foncières reste cependant en retrait des préoccupations, leur rôle dans les questions de l'environnement et des services de base (accès à l'eau et santé notamment) étant mieux traité dans les littératures anglophone et francophone.

Ces résultats montrent bien la prégnance d'un système de gestion coutumière de la terre en zone cotonnière comme l'ont déjà noté plusieurs auteurs (Mathieu et Freudenberger, 1998; Chauveau, 1998 ; Lavigne, 1998 ; Benjaminsen, 2002, Leroy et al. 2006). Les ressources de propriété communautaire peuvent être définies simplement comme celles qui appartiennent à une communauté, même si cette possession est implicite et non formalisée (Mathieu et Freudenberger, 1998). Par rapport à ces ressources, tous les membres du groupe (village, lignage, clan fraction nomade) ont les mêmes droits d'usage, à condition de respecter des règles bien définies par le groupe. Ces mêmes conditions d'accès aux ressources, qui semblent comparables dans les pays d'Afrique subsaharienne, est démontrée par Leroy al. (2006) en Namibie. Les règles d'accès et d'usages des ressources naturelles, ainsi que les procédures de sanction, sont définies au niveau de l'ethnie Owambo et consignées dans une "Constitution des Autorités Traditionnelles ». Pour Chauveau (1998), au sein des systèmes coutumiers, il est difficile de délimiter un domaine ou une "logique » autonome par rapport à des logiques sociales qui l'englobent. Il est aussi difficile d'isoler un «droit » de l'ensemble des relations qui caractérisent la position d'un sujet de droit au sein d'un système social local. Les règles foncières sont d'abord des règles sociales utilisées pour caractériser les relations entre individus. 


\section{Enjeux et litiges fonciers}

Aujourd'hui dans les trois villages, plusieurs enjeux existent autour de la terre. Les ressources foncières sont de moins en moins disponibles. De nos jours, à cause de la pression foncière, elle-même liée à la surexploitation agricole des terres et l'augmentation de la population villageoise et du cheptel, la question foncière est l'une des plus préoccupantes dans les trois villages. Ainsi les règles d'accès se complexifient, alors que les contraintes et les conflits potentiels se multiplient.

Les possibilités d'accès sont de plus en plus minces, car comme le dit le chef de village de Nafègué : « ni y a ye dunan ye dugukolo soro, a ya soro dugulenw faara » (l'allochtone peut avoir satisfaction à sa demande de terre lorsque l'autochtone en a suffisamment). Tous les paysans des villages sont actuellement sur des espaces agricoles dont le renouvellement à travers les pratiques de jachère est extrêmement difficile à cause de l'insuffisance des terres. Par contre à Benguéné, les espaces agricoles sont quasi suffisants mais les sols sont pauvres, même parfois rocheux (bèlè dugukolo). Quant à Ziguéna, ce village dispose de beaucoup de terres agricoles riches mais qui subissent d'énormes pressions à cause de la diversité des activités et l'importance de l'élevage. Rochegude (1998) a montré le caractère sensible de la gestion décentralisée, donc de proximité en milieu rural à cause de la diversité des acteurs et des activités. Pour lui les problèmes de cohabitation agriculteurs/éleveurs et la gestion plus large des ressources ne sont pas forcément résolus, compte tenu des antagonismes entre les besoins d'exploitation des ressources et ceux de la préservation

Ainsi, les conflits fonciers potentiels entre les paysans d'un même village ou entre deux villages voisins sont de plus en plus présents ; la terre étant considérée comme une ressource naturelle épuisable dans le sens de Rotillon (2010). Mais les conflits sont circonscrits à cause de l'ancrage des valeurs séculaires de respect, de tolérance et de compréhension mutuelle entre les protagonistes, mais aussi de l'existence des frontières naturelles matérialisées par les marigots sur les quatre points cardinaux comme à Nafègué. A l'intérieur du village, lorsqu’il y a un litige entre les paysans, il est toujours résolu à l'amiable selon les mécanismes traditionnels adossés aux us et coutumes et jalousement défendus par les autorités coutumières.

Généralement l'aplanissement de certains litiges fonciers passe par le dédommagement des paysans lésés. D’une manière générale, les litiges ne font pas encore l'objet de procès en justice. Chauveau (1998), montre que les jugements coutumiers sont argumentés selon des normes sociales plus morales que juridiques. En effet, dans un conflit quelconque (conflit lié au foncier, à l'eau, à la forêt, etc.) en milieu rural africain, les parties opposées sont liées entre elles par des relations de nature très diverses (de parenté, d’alliance ou de clientèle, etc.). Cependant pour Lavigne (1998), ces normes 
et règles ont un caractère évolutif et peuvent connaitre des transformations profondes. Par exemple pour les terres agricoles on constate une tendance à l'individualisation des droits sur l'espace et les ressources avec régression des formes de contrôle communautaire. Benjaminsen (2002) parle d’érosion des normes coutumières.

\section{Conclusion}

Les trois villages (Nafègué, Ziguéna et Benguéné) sont marqués par l'existence d'organisations socioprofessionnelles (coopératives et associations). Celles-ci concernent toutes les catégories sociales y compris les jeunes et les femmes. Ces organisations sociales sont en général indépendantes les unes des autres. Elles nouent cependant des relations dynamiques dans le cadre de leurs activités et du développement local. Les CPC représentent le noyau des organisations. Elles sont en relation avec toutes les autres organisations et les appuient sur divers plans. La jeunesse se met à la disposition de toutes les couches sociales pour assurer les activités physiques d'intérêt public. L'accès à la terre se fait encore selon les valeurs coutumières. La terre n’est pas encore monétisée. Les règles d'accès à la terre varient selon qu'on soit autochtone, allochtone ou femme. Dans aucun des villages, la femme ne peut être propriétaire terrienne et ne n'accède pas directement à la terre. Elle est toujours obligée de passer par personne interposée. Les enjeux du foncier sont de plus en plus grands à cause de la pression anthropique (augmentation de la population paysanne, diversification des activités, etc.). Cette situation augmente les risques de litiges entre les acteurs.

\section{Remerciements}

Les auteurs de cet article tiennent à remercier l'agence française de développement (AFD) qui a financé cette recherche dans le cadre du projet d’amélioration de la productivité dans la zone cotonnière du Mali (PASE II).

\section{References :}

Aubertin, C., et Pinto, F., (2006). Les paysans: Figure emblématique du développement durable?, in «Le retour des paysans à l'heure du développement durable, Auclair L., Aspse C., Baudot P. (dir.), éd. IRD, Ecologie humaine, Edition Edisud, p.15-29.

Auclair, L., Aspe, C., Baudot, P., (2006). Le retour des paysans à l'heure du développement durable, SEH, éd. IRD, Ecologie humaine, Edition Edisud, Aix en Provence, 360p.

Benjaminsen, T.A., (2002). Transactions foncières et appropriation de la rente foncière dans la zone cotonnière du Mali, European Journal of Development Research, Volume 14, number 2, p.129-152. 
Bertrand, M., (2001). Femmes et marchés fonciers urbains : mesures et déterminants d'une percée à Bamako, Mali, Autrepart (19), p. 29-48

Bozon M., Locoh Th. (dir.) (2000), Rapports de genre et Questions de population, II. Genre, population et développement, Dossiers et recherches, 85, août, Paris, Ined.

Canet, R., et Audet, R., (2005). La société civile à l’Organisation mondiale du commerce : vers une gouvernance plus équitable ?, in «Autrepart $n^{\circ} 35$, Les ONG à l'heure de la bonne gouvernance », éd. IRD, p.161-176.

Chauveau, J.P., (1998). La logique des systèmes coutumiers, in «Quelques politiques foncières pour l’Afrique rurale ?, réconcilier pratiques, légitimité et légalité », Lavigne D.P., (dir.), éd. Karthala-Coopération française, p.6675.

Chauveau, J.P., (1998). Quelle place donner aux pratiques des acteurs ?, «Quelques politiques foncières pour l'Afrique rurale ?, réconcilier pratiques, légitimité et légalité », Lavigne Delville P. (dir.), éd. Karthala-Coopération française, pp.36-39.

Goislard, C., et Djiré, M., (2007). Accès à l’information juridique, aux institutions et procédures légales : Quelle sécurisation foncière pour les ruraux pauvres au Mali ? Etude de cas dans le sud malien, l’Organisation des Nations-Unies pour l'Alimentation et l'Agriculture (FAO), Programme de Soutien aux Moyens d'Existence (LSP), rapport final, 97p.

Lavigne, D.P., (1998). Privatiser ou sécuriser, in quelques politiques foncières pour l'Afrique rurale ?, réconcilier pratiques, légitimité et légalité, Lavigne D.P., (dir.), éd. Karthala-Coopération française, pp.28-35

Leroy, M., Mathieu, G., Méline, V., Moulin, C.H., (2006). La gestion communautaire comme principe de gestion environnement en Namibie (Nord-central) : évaluer les dispositifs pour éviter les mots d’ordre, in « Le retour des paysans à l'heure du développement durable », Auclair L., Aspse C., Baudot P. (dir.), éd. IRD, Ecologie humaine, Edition Edisud, p275-298. Mathieu, P., et Freudenberger M., (1998). La gestion des ressources de propriété communautaire, in «Quelques politiques foncières pour l'Afrique rurale ?, réconcilier pratiques, légitimité et légalité », Lavigne D.P., (dir.), éd. Karthala-Coopération française, p.101-113.

Moulaye, Z., (2005). Gouvernance démocratique de la sécurité au Mali, un défi du développement durable, Imprim Color, Friedrich Ebert Stiftung, Bamako, 197p.

Rochegude, A., (1998). Les instances décentralisées et la gestion des ressources renouvelables, Quelques exemples en Afriques francophone, in «Quelques politiques foncières pour l’Afrique rurale ?, réconcilier pratiques, légitimité et légalité », Lavigne DP., (dir.), éd. Karthala-Coopération française, p.403-421 
Rotillon, G., (2010). Economie des ressources naturelles, Nouvelle Edition, Ed., la découverte, Paris, 125p.

Wanlin, P., (2007). L'analyse de contenu comme méthode d'analyse qualitative d'entretiens : une comparaison entre les traitements manuels et l'utilisation de logiciels, Recherches Qualitatives - Hors-Série - Numéro 3, Actes du colloque bilan et prospectives de la recherche qualitative, p.243272. 